\title{
Introduction au numéro spécial sur le développement des compétences de recherche en enseignement des mathématiques
}

\author{
Igor' Kontorovich • Peter Liljedahl
}

Published online: 19 April 2018

(C) Ontario Institute for Educational Studies (OISE) 2018

"Nos pratiques actuelles au doctorat en éducation sont en fait un mélange de méthodes traditionnelles, de répétition de la manière dont nous-mêmes avons été formés, de commodité administrative et d'inertie. Nous ne soumettons pas nos programmes aux différentes innovations audacieuses ou aux épreuves expérimentales et critiques que nous prétendons valoriser dans nos propres travaux de recherche. » (Shulman 2010, p. 9).

La décision de consacrer un numéro spécial de cette revue scientifique au développement de compétences de recherche en enseignement des mathématiques ne va pas de soi. En effet, certains lecteurs critiques pourraient immédiatement se demander si le sujet mérite un tel niveau d'attention de la part de notre communauté de chercheurs en enseignement des mathématiques. Un coup d'œil rapide aux travaux de recherche de la communauté dans son ensemble (par exemple les revues scientifiques, les publications, les sites web de différents congrès, les programmes universitaires, etc.), révèle que les compétences collectives en recherche se portent probablement mieux que jamais auparavant. D'autres lecteurs pourraient estimer avec raison que la question des compétences en recherche se situe plus naturellement dans la catégorie des études en enseignement supérieur. S'il est vrai que la publication de ce numéro de la RCESMT illustre le fait que tout le monde ne partage pas nécessairement ces opinions contraires, nous considérons la critique comme une bonne façon d'aborder le sujet et d'y réfléchir. De plus, nous estimons qu'il est nécessaire de fournir une partie des réponses aux éventuelles critiques pour établir les justes bases sur lesquelles se fondent les articles présentés dans ce numéro.

En tant que discipline récente qui cherche encore son identité (voir par exemple Fried et Dreyfus 2014), l'enseignement des mathématiques élargit systématiquement ses horizons, affine constamment ses méthodes de recherche et approfondit de plus en plus l'analyse épistémologique de ses propres résultats. De telles tendances sont bienvenues et sont liées à la maturation de notre discipline. Les opinions sur ce point peuvent varier de façon significative, mais on ne saurait nier la prolixité d'idées et de contributions qui

\footnotetext{
I. Kontorovich $(\bowtie)$

Université d'Auckland, Auckland, New Zealand

e-mail: i.kontorovich@auckland.ac.nz

P. Liljedahl

Université Simon Fraser, Burnaby, BC V5A 1S6, Canada
} 
sont proposées presque quotidiennement. Dans de nombreux cas, ces idées et contributions surpassent leurs prédécesseurs dans leur niveau de raffinement et d'incommensurabilité les uns par rapport aux autres. La situation s'avère particulièrement difficile pour les nouveaux arrivants, qui doivent s'y retrouver dans la vaste production que constituent les études du passé et du présent sans pour autant être en mesure de comprendre les règles, souvent non articulées, qui régissent la discipline. Dans cette situation, des attentes visant à proposer de nouvelles interprétations dans un ensemble de connaissances en telle rapidité d'évolution place la barre particulièrement haut. Nous espérons que les lecteurs seront d'accord pour dire que l'intégration des nouveaux venus dans notre communauté de recherche constitue aujourd'hui un défi plus grand que jamais.

S'il est vrai que l'argument invoqué plus haut s'applique également à d'autres communautés de recherche, essayons de le considérer comme partie intégrante d'un ensemble de défis et de possibilités qui définissent tout champ d'études. Par exemple, notre discipline est issue d'une des plus anciennes qui soient. Cependant, elle provient avant tout de la conviction profonde qu'il vaut la peine d'analyser l'apprentissage des mathématiques (et, plus tard, son enseignement) d'une façon qui, rétrospectivement, peut être qualifiée de recherche disciplinée (par exemple, Cronbach et Suppes 1969). (De notre point de vue, le débat sur les façons de présenter cette recherche reste encore ouvert.) Ainsi, la recherche et ses contreparties pratiques sont étroitement liées à l'enseignement des mathématiques, mais cette dernière discipline est beaucoup plus inflexible. En quoi ce recul historique est-il pertinent pour l'intégration des nouveaux chercheurs? Plus souvent qu'autrement, nos nouveaux arrivants proviennent d'autres disciplines qui ont leurs propres traditions, ou encore ils ont déjà une certaine expérience pédagogique, sans oublier leur parcours personnel comme apprenants des mathématiques. Il va de soi que les études en Enseignement supérieur peuvent donner aux nouveaux venus des connaissances fort utiles pour la recherche dans notre discipline. Mais toutes les communautés de recherche n'ont pas comme nous la chance d'attirer une telle variété d'aspirants chercheurs, c'est pourquoi nous estimons qu'il existe un besoin stratégique de développer des interprétations et connaissances spécifiques sur les façon dont les compétences de recherche de nos nouveaux arrivants peuvent être mises au service de l'enseignement des mathématiques au sens large. Avec Shulman (2010), nous proposons que ces interprétations et connaissances soient poussées par la recherche et la pratique afin d'être à la hauteur des standards de qualité que nous valorisons dans nos propres travaux de recherche.

Ce numéro spécial est la conséquence logique du discours actuel sur la formation des chercheurs en didactique des mathématiques. Traditionnellement, dans les cours, dans la supervision et dans les activités de mentorat, ce discours fait partie des pratiques universitaires quotidiennes (par exemple, Schoenfeld 2008). Pourtant, dans les deux dernières décennies, nous avons pu constater que le discours allait au-delà des départements et des facultés pour devenir une priorité collective de la communauté internationale en enseignement des mathématiques. Cette priorité se manifeste par le biais de congrès spéciaux sur les programmes de doctorat (par exemple celui de l'Association of Mathematics Teacher Educators, en 2003), des activités visant à rassembler des jeunes chercheurs provenant de pays différents (par exemple les écoles d'été de la YERME), ainsi que les « journées débuts de carrière » qui précèdent de nombreux congrès dans notre discipline. La forme du discours en recherche est encore en phase de développement (voir, par exemple, Boaler et al. 2003; Kontorovich 2015, 2016; Nardi 2015), et ce numéro spécial se veut une contribution à ce développement. En effet, il est plutôt paradoxal que ce discours fasse l'objet de si peu de recherches, étant donné son ubiquité dans notre communauté et le nombre important de personnes potentiellement intéressées à ces recherches à l'échelle globale (étudiants, superviseurs, administrateurs, concepteurs de programmes). Pour cette raison, nous espérons que dans un proche avenir la recherche en apprentissage et en enseignement des mathématiques puisse faire l'objet d'études théoriques et empiriques dans notre communauté de recherche. Les six articles contenus dans ce numéro ouvrent la voie à ces études futures.

Le numéro présente une série d'études menées au Canada, en Israël, en Italie, en Turquie et aux ÉtatsUnis. Bien que les traditions de recherche et les communautés de chercheurs en enseignement des 
mathématiques varient considérablement entre ces différents pays, les études présentées ici peuvent aisément être séparées en trois groupes. Le premier comprend les travaux d'Igor' Kontorovich et Annette Rouleau d'une part, et de Chiara Andrà et Domenico Brunetto d'autre part, dont les études analysent comment les nouveaux chercheurs provenant d'autres disciplines font leurs premiers pas en recherche sur l'enseignement des mathématiques. Kontorovich et Rouleau centrent leur attention sur les enseignants en fonction qui poursuivent également des études de maîtrise en didactique des mathématiques et qui recueillent des données pour leur premier projet de recherche. Cette étude analyse en détails les rapports d'autoévaluation écrits par les enseignants-chercheurs au sujet de situations dans lesquelles les répondants ont des difficultés ou des idées erronées en mathématiques. Ces situations se sont avérées particulièrement tendues pour les enseignants-chercheurs en raison de ce que les auteurs décrivent comme une sorte de compétition entre les deux identités professionnelles: celle de l'enseignant, dont le rôle est d'aider la personne qui affronte un problème mathématique, et celle du chercheur, qui (du point de vue d'un néophyte) doit simplement prendre une photographie « objective » des conceptions mathématiques de ses répondants.

Andrà et Brunetto concentrent leur analyse sur un étudiant au début de ses études de doctorat, qui provient du département des mathématiques, qui participe à son premier congrès en enseignement des mathématiques et qui est co-auteur de son premier article scientifique avec sa directrice de thèse. La directrice est elle-même à ces débuts puisqu'il s'agit de son premier étudiant au doctorat. Grâce à l'analyse des récits autobiographiques de l'étudiant et de sa directrice, l'étude présente une série de problèmes, de tensions et de contradictions que les deux sujets ont dû affronter. Pour les auteurs, cet éventail d'émotions représente autant d'occasions de réflexion et de modification des pratiques.

Dans les deux études suivantes, Çiğdem Haser et Peter Liljedahl centrent leur attention sur des étudiants de deuxième et troisième cycles en didactiques des mathématiques et analysent leurs idées et opinions quant au fait de devenir des chercheurs autonomes. Haser se penche sur les idées des étudiants au doctorat (qui s'orientent vers une carrière universitaire) au sujet de ce qui caractérise la recherche indépendante en enseignement des mathématiques et si ces caractéristiques s'appliquent à eux. Dans son analyse des résultats, Haser souligne le rôle significatif de la communauté des pairs en ce qui concerne la formation des expériences et les compétences personnelles de recherche. Il est intéressant de noter que, dans certains cas, les communautés avaient été formées par les étudiants eux-mêmes.

L'étude réalisée par Liljedahl analyse comment les étudiants changent leur façon de concevoir la recherche à mesure qu'ils progressent dans leur programme. L'étude fournit un rapport détaillé des similarités et des différences dans les façons de penser des étudiants de maîtrise ou de doctorat, et à différentes étapes de leurs études. Les résultats de Haser sur l'importance de la communauté de chercheurs émergent également dans cette étude. Liljedahl, insiste à son tour sur le fait qu'il ne faut pas entendre cette communauté comme une équipe de pairs qui travaillent à un même projet de recherche, mais bien comme un réseau sécuritaire où l'on peut partager et échanger avec d'autres personnes qui se trouvent dans une situation comparable.

Les deux derniers articles, l'un de Robert Reys et l'autre de Boris Koichu et Alon Pinto, nous rappellent les engagements plus larges qui vont de pair avec la recherche universitaire en enseignement des mathématiques. Sur la base de ses quatre décennies d'expérience de supervision, et partant des données statistiques provenant des programmes de doctorat aux États-Unis, Reys partage sa vision d'un parcours idéal qui permettrait de devenir enseignant ou didacticien des mathématiques. Ce parcours suggère des connaissances et expériences qui méritent d'être explorées, en tenant compte du fait que la recherche universitaire n'est qu'une des possibilités de carrière qui s'offrent aux étudiants au terme de leurs études de doctorat.

Pour Koichu et Pinto, les études de deuxième et troisième cycles ne sont pas la seule façon d'acquérir des compétences de recherche pour les enseignants et chercheurs en mathématiques. L'article présente un cadre théorique-organisationnel visionnaire dans lequel les chercheurs et les enseignants en fonction établissent des partenariats d'envergure et se penchent sur des questions qui intéressent les deux profils. De nombreux exemples illustrent les prémisses et heuristiques de ce cadre, visant à la création de partenariats productifs. 
Selon les auteurs, les compétences de recherche des enseignants peuvent aisément aller de pair avec leur travail de collaboration avec les chercheurs en enseignement des mathématiques.

Les lecteurs se rendront vite compte combien les études présentées ici sont différentes entre elles, en termes d'approches, de méthodes et de fondements théoriques. En tant que rédacteurs, nous estimons qu'une telle diversité est nécessaire lorsqu'il s'agit d'aborder un phénomène complexe. En effet, chacune de ces études, ainsi que ce numéro spécial dans son ensemble, illustrent on ne peut plus clairement la complexité du sujet que nous traitons. Nous espérons que cette complexité sera reconnue des lecteurs, et qu'ils sauront discerner les idées importantes pour eux non seulement dans une perspective de recherche, mais aussi du point de vue pratique. Par ailleurs, étant donné que de nombreuses personnes qui ont été les piliers de notre discipline sont maintenant à la retraite ou, malheureusement, nous ont quittés, son avenir dépend des compétences de recherche des nouveaux venus actuels et futurs.

\section{Références}

Association of Mathematics Teachers Education (2001). Principles to guide the design and implementation of doctoral programs in mathematics education. AMTE Publications. Repris le 2 février 2015 à partir du site http://amte. net/publications.

Boaler, J., Ball, D. L., et Even, R. (2003). Preparing researchers for disciplined inquiry: Learning from, in, and for practice. In A. Bishop et J. Kilpatrick (Éd.), International Handbook of Mathematics Education (pp. 491-521). Dordrecht : Kluwer.

Cronbach, L. J., et Suppes, P. (Éd.). (1969). Research for tomorrow's schools : Disciplined inquiry for education. New York : Macmillan.

Fried, M. N., et Dreyfus, T. (2014). Mathematics and mathematics education : Searching for common ground. Dordrecht Heidelberg, New York London : Springer.

Kontorovich, I. (2015). Learning from the experts in mathematics education research. Far East Journal of Mathematics Education, 15(1), 35-56.

Kontorovich, I. (2016). Theoretical framework of researcher knowledge development in mathematics education. International Journal of Education in Mathematics, Science and Technology, 4(2), 101-111.

Nardi, E. (2015). "Not Like a Big Gap, Something We Could Handle": Facilitating shifts in paradigm in the supervision of mathematics graduates upon entry into mathematics education. International Journal of Research in Undergraduate Mathematics Education. Publié en ligne.

Schoenfeld, A. H. (2008). Research Methods in (Mathematics) Education. In L. English, Handbook of international research in mathematics education, $2^{\mathrm{e}}$ édition (pp. 467-519). New York : Routledge.

Shulman, L. S. (2010). Doctoral education shouldn't be a marathon. The Chronicle of Higher Education : The Cehronicle Review. 